Revue d'histoire de l'Amérique française

REVUE D.HISTOIRE DE L'AMÉRIQUE FRANÇAISE

\title{
Revue d'histoire des Colonies (Paris, Au siège de la Société : 43, rue Cambon). Cette importante revue a recommencé à paraître en 1947. Le fascicule — le seul de toute l'année - est de 150 pages
}

\section{Lionel Groulx}

Volume 2, numéro 4, mars 1949

URI : https://id.erudit.org/iderudit/801517ar

DOI : https://doi.org/10.7202/801517ar

Aller au sommaire du numéro

Éditeur(s)

Institut d'histoire de l'Amérique française

ISSN

0035-2357 (imprimé)

1492-1383 (numérique)

Découvrir la revue

Citer ce compte rendu

Groulx, L. (1949). Compte rendu de [Revue d'histoire des Colonies (Paris, Au siège de la Société : 43 , rue Cambon). Cette importante revue a recommencé à paraître en 1947. Le fascicule - le seul de toute l'année - est de 150 pages]. Revue d'histoire de l'Amérique française, 2(4), 608-612.

https://doi.org/10.7202/801517ar d'utilisation que vous pouvez consulter en ligne. 
Revue d'histoire des Colonies (Paris, Au siège de la Sociéte: 43, rue Cambon). Cette importante revue a recommencé à paraître en 1947. Le fascicule - le seul de toute l'année - est de 150 pages.

Nous recommandons la lecture de deux articles: "Une tentative d'hégemonie française en Amérique du Nord, 1689", par Robert Le Blant, p. 22-30; et "Les Travaux d'histoire sur Saint-Domingue, 1938-1946, Essai de mise au point", par G. Debien, p. 31-86. Dans ce dernier article, M. Debien, - qui est, comme l'on sait, l'un des membres-correspondants de notre Institut - tente "une revue générale des travaux parus pendant la guerre sur l'histoire de Saint-Domingue". Pièce d'importance pour l'histoire des Antilles françaises.

"Une tentative d'hégémonie française en Amérique du Nord, 1689" n'est rien d'autre, on l'aura deviné, que le projet de conquête, de la Nouvelle-York par une attaque combinée sur terre et sur mer, de la flotte française commandeө par La Caffinière, destinée au blocus du port de Manhattan et d'une troupe de 1,600 miliciens et soldats dirigée par Frontenac sur Orange, puis sur Manhattan. M. Le Blant s'en prend à cette opinion de Garneau, (Histoire du Canada, nouvelle édition, t. I, p. 367), qu'en 1689, 
"les colons furent entraînês dans une guerre dont l'objet leur était étranger". Selon M. Le Blant, Garneau aurait prouvé "son ignorance à peu près complète de la question iroquoise". L'Iroquois constituait, pour le Canada, le suprême péril. En 1689 la colonie ne se pouvait liberer de ce cauchemar que par la stricte neutralité des colons anglais ou par la conquête de la NouvelleYork. (En passant, M. le Blant nous permettra de lui faire observer que l'édition de Garneau à laquelle il nous renvoie, est celle de 1913 publiée à Paris et que la désignation "nouvelle édition" pourrait prêter à équivoque, puisque cette "nouvelle édition" est en réalité la cinquième de Garneau, et que depuis, il en a paru une sixième au Canada). Nous ne discuterons pas l'opinion de Garneau qui nous paraît assez peu fondée. De toute façon la guerre déclarée en Europe voulait dire la guerre en Amérique. Pour réfuter Garneau, M. Le Blant n'aurait eu besoin que de rappeler l'extraordinaire sursaut de fanatisme protestant provoqué dans les colonies anglaises du sud, par l'avènement de Guillaume d'Orange au trône d'Angleterre. Contre les fonctionnaires du Stuart, aussi rapide qu'un feu de forêt, l'insurrection courut de Boston au Chesapeake. L'appel de New-York aux colonies-sœurs donna lieu à ce qu'on pourrait appeler le premier congrès des États-Unis. Et sans outrer plus qu'il ne faut le rôle des partis de guerre organisés par Frontenac pendant l'hiver de 1689-1690, et qui ont paru à quelques-uns des provocations, c'est un peu comme à une guerre sainte que les colonies du sud se préparèrent à leur campagne contre l'Acadie et le Canada.

Non, M. Le Blant nous donnerait plutôt l'envie de poser des questions comme celles-ci: neutralité perpétuelle des colonies anglaises ou conquêtê de la Nouvelle-York, était-ce bien les seules façons de conjurer le péril iroquois? N'insistons pas sur la neutralité possible des "treize colonies" qui ne pouvait être, pour tout esprit réaliste en Amérique du Nord, qu'une noble chimère. Interrogeons-nous plutôt sur l'aspect pratique de cette conquête de la Nouvelle-York ou de la Nouvelle-Angleterre, projet qui remonte, comme l'on sait, au Père LeJeune, projet que nourrit Talon et que vont reprendre Callières, Frontenac et quelques autres après eux. Négligeons encore si l'on veut, le côté hasardeux de ces projets de guerre, par mer et par terre, combinés de Versailles et dont le succès, comme on l'a bien vu quelquefois, tenait à moins qu'un fil ? Dans ces traversées océaniques de durée si variable, comment organiser de si loin des expéditions qui exigeaient, pour leur réussite, outre la ponctualité précise à quelques rendez-vous, une simultanéité parfaite de mouvements entre forces navales et forces de terre. Ces difficultés paraissaient d'ailleurs à peu près insurmontables à Frontenac qui, en 1689, en avait averti la Cour. Comme il arriva presque toujours, en ces occurrences, la Caffinière partit trop tard de LaRochelle. On commit, du reste, l'erreur de faire voyager Frontenac et Callières, sur l'un des deux vaisseaux de La Caffinière, au lieu de les envoyer de l'avant pour préparer l'expédition de terre. Après une traversée de 52 jours, Frontenac et Callières n'atteignirent Chedabouctou que le 12 septembre. C'était déjà bien tard, si tard que Frontenac ne pourra trouver, même à Percé, de vaisseaux 
pộcheurs pour hâter l'arrivée à Québec de Callières. Le gouverneur n'atteignit lui-même la capitale que le 12 octobre au soir. Ni Denonville ni Champigny ne s'y trouvaient, retenus à Montréal par l'urgence des affaires. Comment, à cette saison de l'année et dans l'état déplorable de la colonie, et face à l'hostilité acharnée de l'Iroquois - l'on était au lendemain du massacre de Lachine - comment, en quelques jours, mobiliser un millier de miliciens dispersés sur une distance de plus de 180 milles, ramasser des vivres et des munitions, organiser des oonvois pour une descente du SaintLaurent jusqu'à Manhatte? Ces Messieurs de la Cour avaient-ils calculé, qu'en raison des distances et des hostilités à affronter le long de la route, ces sortes d'excursions ne se pouvaient préparer, ni en quelques jours, ni en quelques semaines? L'expédition par terre était ratée. Nul besoin de rappeler ce qu'il advint de $M$. de La Caffinière et de ses vaisseaux.

$\mathrm{Du}$ reste, on pouvait peut-être prendre Orange et Manhatte. Autre chose serait de les garder. Puisqu'on se limitait à la prise de la NouvelleYork, comment défendre et conserver longtemps cette enclave, située comme elle le serait, entre le pays des Iroquois et la Nouvelle-Angleterre? Le roi voulait que, la conquête terminée, on expulsât les suspects, et que le peuplement de la région fût confiée aux troupiers et aux habitants du Canada. Mais comment prélever ces nouvelles équipes de défricheurs ou d'agriculteurs dans une colonie dont la suprême misère se ramenait à un manque de bras, à un peuplement déficient, et qui, par sa faiblesse en hommes, se trouvait incapable d'inspirer le respect à ses plus proches voisins, les Iroquois? Nous touchons ici au seul moyen pratique à l'époque de protéger efficacement le Canada et contre l'Iroquois et contre l'Anglais: le peupler, le peupler à fond, avec méthode et persévérance. Le péril iroquois n'eût point existé pour un Canada, peuplé seulement au double de ce qu'il l'était vers 1690 et qui, par sa seule milice, eût pu mettre sur pied, autant de combattants que les cinq Cantons. Le péril iroquois est né, pour la grande part, de la faiblesse de la colonie et a duré pour la même cause. A-t-on jamais compris ces simples vérités à la Cour de France ? Garneau eût pu adresser à la politique de Louis XIV un reproche beaucoup plus embarrassant, et ce fut précisément, en 1689, trois semaines après la déclaration de guerrê de Guillaume d'Orange, d'avoir manifesté l'intention de vider la Canada, aussitôt que possible, de ses maigres troupes de défense, pour abandonner la Colonie à sa seule milice. '́ Évidemment Sa Majesté ne songeait nullement à opérer ce retrait pendant la guerre; mais la conquête de la Nouvelle-York mise en sécurité, et les besoins du Canada assurés(?), elle espérait bien qu'il se trouverait quelques troupes inutiles, en état d'être renvoyées en France. ${ }^{2}$ Calcul singulier qui révèle par soi-même quelle illusion la Cour entretenait sur l'état des choses en Amérique.

1. "Instructions pour le Comte de Frontenac," 7 juin 1689, Rapport de l'Archiviste de la province de Québec (1927-1928): 7.

2. ibid. 15. 
On objectera que la France elle-même manquait d'hommes pour ses guerres et ses industries et que ses finances ne lui permettaient plus de renouveler l'effort de 1660 à 1672. Eût-il été besoin d'un si grand effort? Ceux qu'on a appelés les "engagés" ne manquaient pas dans la métropole; et chacun sait qu'ils prenaient la route de bien d'autres pays que le Canada et que les autres possessions françaises d'outre-mer. Une simple et persévérante mise en vigueur des règlements pour le transport des "engagés" sur les vaisseaux faisant voile vers le Canada, et même un peu de dirigisme dans l'orientation de ces "engagés", n'eussent-ils pas suffi à peupler la colonie? Et la merveille, c'est que cette sorte d'émigration n'eût d'aucune façon obéré ni le budget métropolitain ni celui des armateurs, ni même celui des colons canadiens, puisque les colons, toujours en quête de main-d'œuvre, remboursaient aux armateurs les frais de transport, quittes à se rembourser eux-mêmes par les services des "engagés". On objectera peut-être encore que, pour bien fonctionner, ce système des "engagés" eût requis du trésor royal, pour le recrutement des émigrants, quelque forme d'aide aux armateurs. Possible. Mais cette aide eût-elle coûté plus cher que le maintien continuel au Canada de 1,2,00 à 1,500 hommes de troupes, et plus cher aussi que la vorace alliance des Indiens des Lacs?

Dans un peuplement généreux, continu, résidait, et pas ailleurs, l'avenir français du Canada. Il fallait des hommes, beaucoup d'hommes pour exploiter un pays aux dimensions de la Nouvelle-France d'Amérique. Il en fallait beaucoup pour tenir tête aux Iroquois et surtout aux colonies voisines qui se développaient au pas de course. Perdre de vue ces aveuglantes réalités, négliger l'énorme disproportion de forces qui allait s'aggravant chaque jour entre colonies du nord et colonies du sud, se flatter qu'il fût possible d'eviter une catastrophe, quand, en Europe, l'inégalité de forces s'esquissait d'ores et déjà entre les deux métropoles rivales, et qu'ici-même, en Amérique, de puissants intérêts jetaient fatalement colonies anglaises et colonies françaises les unes contre les autres, ce n'était pas seulement une singulière erreur d'optique; c'était, pour le roi de France, comme on l'a dit tant de fois, travailler proprement pour le roi d'Angleterre.

Dans la même Revue d'histoire des colonies, t. XXXV, (1948): 84-113. Premier semestre.

Nous recommandons une autre étude de M. Robert Le Blant: "Les études historiques sur la colonie française d'Acadie, 1603-1713”. M. Le Blant fait la revue critique des sources manuscrites et imprimées de l'histoire acadienne: Archives nationales, Bibliothèque Nationale, Archives du Ministère des Colonies, Archives départementales de la CharenteInférieure, Fonds divers, puis œuvres de Marc Lescarbot, de Champlain, du jésuite Biard, de Nicolas Denys, de Chrestien Leclercq, de Monseigneur de Saint-Vallier, de Diéréville, de Robert Chevalier, dit de Beauchesne, de Robert Challes. Puis encore, dans une troisième partie, revue des 
"Travaux historiques sur l'Acadie" où passent, avec critique souvent sévère, travaux français et travaux anglais et americains, publications de documents. Viennent en procession Lescarbot, Charlevoix, Garneau, Moreau, Rameau, Richard, Lauvrière, Couillard Després, Huguet, l'abbé Maurault. Évidemment, M. Le Blant qui se plaint ailleurs de l'absence de maints ouvrages d'histoire canadienne dans les bibliothèques de France, - surtout parmi les plus récents - ne connaît pas la série des Cahiers des Dix, sans quoi il n'aurait pas fait naître Beauchesne vers 1693, "d'une famille inconnue", alors qu'il a été bien établi par Aegidius Fauteux, Cahiers des Dix, 2 (1937): 20, 24, "que le héros de LeSage est bel et bien né à la Pointeaux-Trembles près Montréal de Jacques Chevalier et de Jeanne Villain et qu'il y a été baptisé le 23 avril 1686". Selon M. Le Blant, "étant donnée la confusion créée par les historiens... une nouvelle rédaction de l'histoire de la colonie française d'Acadie devrait être entreprise d'après les sources..."

Lionel Groulx, ptre 